\title{
Median Arcuate Ligament Syndrome: An Exceptional Cause of Abdominal Pain
}

\author{
Satilmis Bilgin*, Gulali Aktas, Ozge Kurtkulagi, Tuba Taslamacioglu Duman, Burcin Meryem Atak, \\ Gizem Kahveci, Mehmet Zahid Kocak, Haluk Savli
}

Department of Internal Medicine, Abant Izzet Baysal University Hospital, Bolu, Turkey.

\begin{abstract}
Median Arcuate Ligament Syndrome (MALS) is caused by the compression of celiac artery by median arcuate ligament and may cause serious symptoms; such as abdominal pain and weight loss. Here we present a 56 year old man with MALS, whom suffered of postprandial abdominal pain, weight loss, nausea and vomiting. Laparoscopic surgery revealed his symptoms after MALS diagnosis was established. In conclusion, MALS should be considered in the differential diagnosis of patients with complaints of postprandial abdominal pain, nausea and weight loss. Laparoscopic surgery could be as successful as other treatment options in these patients.
\end{abstract}

Keywords: Median arcuate ligament syndrome, Postprandial abdominal pain, Laparoscopic surgery, Nausea, Celiac disease, Coronary artery disease.

\section{INTRODUCTION}

At the level of T12/L1 vertebra, a fibrous band, namely, median arcuate ligament (MAL) connects the diaphragmatic crus on both sides of the aorta [1]. Low-level localization of this ligament causes stenosis in the proximal celiac artery, leading to ischemic symptoms known as median arcuate ligament syndrome (MALS) or celiac artery compression syndrome [2]. The syndrome was initially described in 1965 [3]. Its prevalence is about 2 in 100000 population of which only few have significant obstruction that cause hemodynamic instability [1]. It is more common females aged between 20 to 40 years.

Symptoms of MALS develop by reduction of the blood flow of the gastrointestinal tract due to compression of the proximal celiac artery at the aortic outlet. Despite most of the subjects are asymptomatic, epigastric pain that increase during expiration, nausea and vomiting after oral nutrition and weight loss are cardinal features in symptomatic patients $[4$, $5]$.

In this report, we aimed to present a patient that complained of postprandial abdominal pain, and nausea whom consequently diagnosed with MALS.

\section{CASE REPORT}

A 56-year-old male was admitted to our clinic with a 3 months of history of abdominal pain and nausea that increased after eating which had worsen progressively. The pain was localized to the epigastric and periumbilical region and was exacerbating several hours after meals. His appetite was decreased in the last few days because of the fear of post-eat-

*Address correspondence to this author at the Department of Internal Medicine, Abant Izzet Baysal University Hospital, Bolu, Turkey.

Email: drsatilmisbilgin@gmail.com ing pain. He stated that he had lost weight since his symptoms developed. A proton pump inhibitor and a spasmolytic were prescribed by his regular family physician two months ago, however, his symptoms did not relieved.

He had a history of celiac disease and coronary artery disease for 2 years and undergone coronary artery bypass graft and cholecystectomy surgery 5 years ago. His medications include had been receiving metoprolol, clopidogrel and atorvastatin which he was being used for the last 2 years.

He was well appeared on physical examination. Systolic and diastolic blood pressure of the patient were $115 \mathrm{mmHg}$ and $70 \mathrm{mmHg}$, respectively. His heart rate was 76 per minute, body temperature was 36.8 celcius degree and respiratory rate was 16 per minute. Thoracotomy scar was notable on sternum. Operation scar of cholecystectomy was noted in abdominal examination. On palpation, mild tenderness on epigastric region was detected. The physical examination was otherwise normal.

In laboratory analyses, hemogram, routine biochemistry, complete urinalysis and C-reactive protein levels of the patient was all in normal range.

Alimentation through oral route was initiated since he had not developed vomiting or nausea during hospital care. Possible causes of postprandial abdominal pain were investigated.

Direct $\mathrm{X}$ ray film of the abdomen was non-diagnostic. Abdominal ultrasonography was showed no pathological images other than grade 1 hepatosteatosis. Contrast enhanced abdominal computerized tomography $(\mathrm{CT})$ revealed reduction in the calibration of celiac artery at the level of MAL and post stenotic dilatation (Figs. 1, 2). A diagnosis of MALS was established based on these findings. 


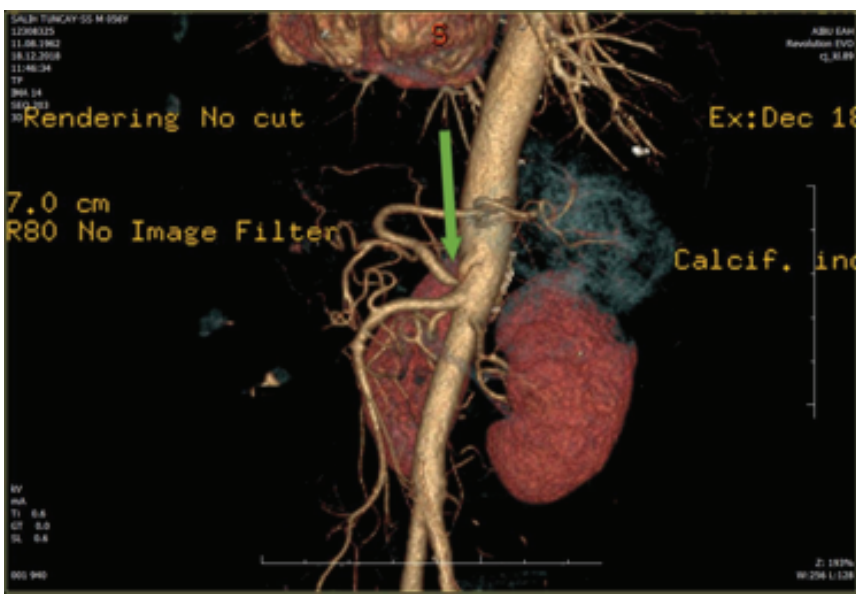

Fig. (1). Three Dimensional Contrast Enhanced Computerized Tomography Shows MAL that Obstructing Celiac Artery (green arrow).

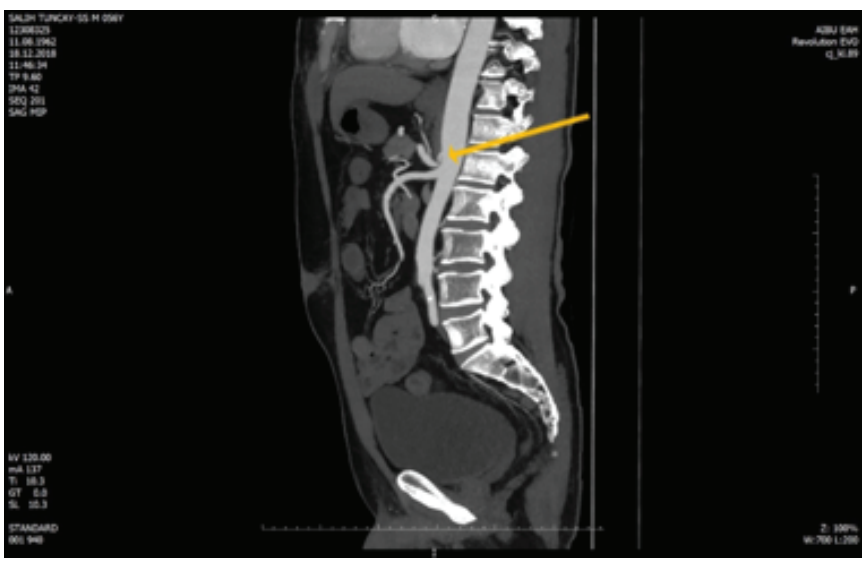

Fig. (2). Sagittal Axis Contrast Enhanced Computerized Tomography Shows MAL that obstructing Celiac Artery (yellow arrow).

An interventional radiology consultation was obtained for stenting the celiac artery in the treatment of MALS, however, since the obstruction was critical, stenting of the artery was considered as impossible, therefore, a surgical consultation was obtained. The MAL was decompressed by laparoscopic surgery. The post-operative period was uneventful and the patient was discharged on $2^{\text {nd }}$ day after surgery. At $30^{\text {th }}$ day, he was free of symptoms on clinic examination.

\section{DISCUSSION}

The MALS is a vascular pathology that usually occur in proximal parts of celiac artery by the compression of MAL. The degree of compression maximizes during deep expiration. Sometimes the external pressure to the artery may lead to complete obstruction of the vascular lumen. Postprandial abdominal pain, weight loss, nausea and vomiting may develop and indicate mesenteric ischemia. Present patient had weight loss, nausea and abdominal pain and fear of post-nutritional pain caused denial of oral feeding.

Murmur in the epigastric region, which is prominent during expiration is an important clinical finding in patients with MALS. However, aortic aneurysm and retroperitoneal fibrosis may also have similar findings in physical examination, and it should be listed in differential diagnosis [6]. In present case, imaging modalities were helpful in establishing diagnosis and ruling out the differentials.

There are two main theories in the development of symptoms and clinical findings in MALS. First, mesenteric ischemia due to obstruction of the celiac artery. Second, neurogenic stimulation due to compression of celiac ganglion and celiac plexus [7-9].

Magnetic resonance imaging, color Doppler ultrasonography, $\mathrm{CT}$ angiography and conventional angiography all can be used in the diagnosis of MALS [7,10]. We preferred three dimensional $\mathrm{CT}$ and $\mathrm{CT}$ angiography in the diagnostic process of the present case.

The treatment of MALS is the surgical removal of the pressure by cutting the obstructing part of MAL. However, endovascular methods that increase celiac blood flow by percutaneous angiographic methods can be applied in selected cases in which conventional treatment has failed [11]. Authors suggest that both laparoscopic surgery, open surgery and endovascular interventions were useful in the treatment of MALS [12]. The main purpose of surgery is to prevent the degeneration of the celiac artery by eliminating the obstruction as soon as possible caused by MAL [13]. In present case, stenting of celiac artery considered as impossible since the obstruction in the artery was serious, therefore, laparoscopic surgery was preferred. Surgery was successful, so, his complaints were resolved immediately.

In conclusion, patients with complaints of postprandial abdominal pain, nausea and weight loss should be evaluated for possible MALS. Laparoscopic surgery could be as successful as other treatment options in these patients.

\section{AUTHORS' CONTRIBUTION}

All authors have equally contributed.

\section{CONFLICT OF INTEREST}

Declared none.

\section{ACKNOWLEDGEMENTS}

Declared none. 


\section{REFERENCES}

[1] Schiappacasse G, Maldonado I, Varela C, Muller CK, Ortiz VJ. Median arcuate ligament syndrome. Rev Chil Radiol 2014; 20: 149-55. DOI: 10.4067/S0717-93082014000400005

[2] Aschenbach R, Basche S, Vogl TJ. Compression of the celiac trunk caused by median arcuate ligament in children and adolescent subjects: Evaluation with contrast-enhanced MR angiography and comparison with Doppler US evaluation. J Vasc Interv Radiol 2011; 22: 556-61. DOI: $10.1016 /$ j.jvir.2010.11.007

[3] Harjola PT. A rare obstruction of the coeliac artery. Report of a case. A Ann Chir Gynaecol Fenn 1963; 52: 547-50.

[4] Horton KM, Talamini MA, Fishman EK. Median arcuate ligament syndrome: Evaluation with CT angiography. Radiographics 2005; 25: 1177-82. DOI: 10.1148/rg.255055001

[5] Alehan D, Dogan OF. Pediatric surgical image. A rare case: Celiac artery compression syndrome in an asymptomatic child. J Pediatr Sur 2004; 39: 645-7.

DOI: 10.1016/j.jpedsurg.2003.12.037

[6] Marcoccia A, Zippi M, Bruni A, et al. Chronic abdominal pain associated with intermittent compression of the celiac artery. Minerva Gastroenterol Dietol 2007; 53: 209-13.

[7] Cina CS, Safar H. Successful treatment of recurrent celiac axis compression syndrome. A case report. Panminerva Medica 2002; 44: 69-72.

[8] Bech FR. Celiac artery compression syndromes. Surg Clin North Am 1997; 77: 409-24.

DOI: $10.1016 / \mathrm{S} 0039-6109(05) 70558-2$

[9] Tribble CG, Harman PK, Mentzer Jr RM. Celiac artery compression syndrome: Report of a case and review of current opinion. Vasc Surg 1986; 20: 120-9. DOI: $10.1177 / 153857448602000211$

[10] Kopecky KK, Stine SB, Dalsing MC, et al. Median arcuate ligament syndrome with multivessel involvement: diagnosis with spiral CT angiography. Abdominal Imaging 1997; 22: 318-20. DOI: $10.1007 / \mathrm{s} 002619900199$

[11] Hongsakul K, Rookkapan S, Sungsiri J, et al. A severe case of median arcuate ligament syndrome with successful angioplasty and stenting. Case RepVasc Med 2012; 2012: 129870 DOI: $10.1155 / 2012 / 129870$

[12] Duncan AA. Median arcuate ligament syndrome. Curr Treat Options Cardiovasc Med 2008; 10: 112-6.

DOI: 10.1007/s11936-008-0012-2

[13] Mucs M, Székely I, Illés I, et al. Celiac artery compression syndrome-stepchild of abdominal angina? Orv Hetil 2007; 148: 1763-6. DOI: 10.1556/oh.2007.28126 